\title{
Perfil clínico e epidemiológico de cães epilépticos atendidos no hospital veterinário da UFRRJ
}

\section{Clinical and epidemiological profile of epileptic dogs referred to the Veterinary Hospital of UFRRJ}

\author{
Luiz Felipe Castro Graeff Vianna, ${ }^{*}$ Kátia Borges Vahia**
}

\begin{abstract}
Resumo
O presente trabalho caracteriza o perfil clínico e epidemiológico de cães epilépticos atendidos no Hospital Veterinário da Universidade Federal Rural do Rio de Janeiro e avalia também, a eficiência clínica do tratamento preventivo das convulsões. Os resultados mostram que a maioria dos animais é de machos não castrados, entre as idades de um a cinco anos, aparecendo àqueles sem raça definida em primeiro lugar. $O$ tipo de crise mais observado foi o da crise generalizada tônicaclônica associada à perda de consciência, com fases bem definidas de pródromo, aura e pós-ictus. A terapia clínica com o uso do fenobarbital mostrou-se bastante eficaz no controle das crises convulsivas.
\end{abstract}

Palavras-chave: epilepsia, convulsões, cão.

\begin{abstract}
The present study characterize the clinical and epidemiological profile of the epileptic dogs seen by the professionals of the Veterinarian Hospital of Rural Federal University of Rio de Janeiro and also to study the clinic efficiency of the preventive treatment of the convulsions. The majority of the animals were intact males in between one and five years old without defined race. The most common type of crises was the generalized (tonic-clonic crises) related to the loss of conscious, with welldefined stages of the crises prodromal, aura and pos-ictus. The therapy with Phenobarbital turned out to be very effective to the control of the convulsive crises.
\end{abstract}

Keywords: epilepsy, convulsion, dogs.

\section{Introdução}

A epilepsia é uma das mais comuns e importantes doenças do sistema nervoso central (SNC) e vem sendo classificada dentro de dois tipos etiológicos em humanos e cães. A epilepsia idiopática inclui todas as desordens convulsivas que não podem ser associadas com patologia microscópica. Já a epilepsia sintomática inclui todas as desordens originadas a partir de danos definidos, doenças metabólicas ou anormalidades neuroestruturais.

É uma desordem envolvendo potencial risco de vida e ocorre em cães de todas as raças. Estima-se a incidência em torno de 0,5 a $5,7 \%$ com base em dados de hospitais de referência. De todos os cães com presença de sinais neurológicos, aproximadamente $14 \%$ tinham relação com crises convulsivas (Bollinger-Schitz e Kline, 2000).

É muito freqüente que o animal levado a uma consulta devido à ocorrência de crises convulsivas seja avaliado como normal no exame físico e nas análises complementares. Neste caso, a anamnese é o elemento mais importante com o qual contamos para a avaliação (Pelegrino, 1999). segundo Berendt et al. (2002), o diagnóstico da epilepsia é essencialmente clínico, baseado nos registros médicos, nos exames neurológicos e no histórico das crises epilépticas.

Poucos estudos sobre a epidemiologia da epilepsia canina foram publicados durante os últimos anos. Com poucas exceções, as descobertas destes estudos foram baseadas em dados de práticas de referência retrospectivas de hospitais-base. A falta de definições padronizadas e metodologia definida com relação à seleção do paciente, precisão de diagnóstico e classificação das crises, torna difícil a comparação de resultados entre os estudos. Vários investigadores usam diferentes definições de epilepsia, diferentes métodos de averiguação dos casos e diferentes modelos de classificação (Berendt et al., 2002).

Este trabalho utilizou um questionário que procurou ser uma síntese de vários trabalhos realizados por diversos autores (Podell e Fernner, 1993; Koutinas et al., 1994; Podell,1995; Heynold et al., 1997; Famula e Oberbauer, 1998; Jaggy e Bernardini, 1998; Jaggy et al., 1998; Knowles, 1998; Bateman e Parent, 1999; Berendt e Gran, 1999; Lengweiler e Jaggy, 1999; Kathmann et al., 1999; Thomas, 2000; Saito et al., 2001; Berendt et al., 2002) nos quais encontramos indagações múltiplas e distintas entre si, porém todas com o propósito de caracterizar

* Professor titular de Clínica Médica, DMCV, UFRRJ.

** Médica-veterinária autônoma, MS, UFRRJ. 
um perfil epidemiológico da amostra estudada. Em comum, estes questionários procuravam identificar a idade da primeira crise observada, a existência ou não do predomínio de sexo ou raça, a classificação das crises convulsivas ocorridas e, por fim, a eficiência do tratamento clínico.

\section{Material e métodos}

Foram avaliados os dados coletados de 74 cães com histórico de crises convulsivas, atendidos no Hospital Veterinário da Universidade Federal Rural do Rio de Janeiro e por veterinários autônomos, entre janeiro de 2000 a junho 2002. Destes, 50 cães foram considerados epilépticos. A investigação incluiu a coleta de dados a partir de registros hospitalares médicos (SAITO et al., 2001), utilização de um questionário para cada cão, respondido pelo proprietário pessoalmente ou através de telefonemas (Heynold et al., 1997), além de avaliações de exames neurológico e físico.

As convulsões foram classificadas em generalizadas primárias, parciais simples, parciais complexas com ou sem generalização secundária (Berendt e Gram,1999) .

\section{Resultados e discussão}

Houve um predomínio na ocorrência de crises convulsivas em cães sem raça definida (SRD), seguido pelas raças Poodles e Pastor Alemão (Figura 1). Segundo Koutinas et al. (1994), a incidência de epilepsia idiopática (EI) é aproximadamente a mesma entre cães puros e àqueles provenientes do cruzamento de raças de alto risco. Porém, os mesmos autores ressaltam que a incidência em mestiços encontrada na literatura é relativamente baixa, o que contrasta com as nossas observações, decerto, em razão do predomínio de animais SRD em nosso Hospital, assim como a popularidade do Poodle e do Pastor Alemão, frente a outras raças em nosso meio. Com relação ao sexo dos animais acometidos, houve um predomínio em cães machos (64\%) e apenas dois animais eram castrados, um macho e uma fêmea. Alguns autores como Jaggy et al. (1998), Famula e Oberbauer (1998) concluíram que o sexo dos animais tem pouca ou nenhuma importância na transmissão da El. Porém, Podell (1995) e Kathmann et al. (1999) observaram uma incidência significativamente maior em machos do que em fêmeas. Podell e Fernner (1993), em amostra de 20 cães com El, observaram que a ocorrência de El em cães castrados - 0 que foi verificado tanto para machos quanto para fêmeas - foi maior do que a ocorrência em cães não castrados, contrastando com nossa observação. Considerando a idade dos animais à primeira crise convulsiva, os cães da faixa etária de 1 a 5 anos foram os mais acometidos (78,0\%) (Figura 2). Isto está de acordo com Thomas (2000), o qual afirmou que muitos cães com El sofreram suas primeiras crises convulsivas nesta faixa etária. Considerando a atividade do animal durante o surgimento da crise convulsiva, houve uma maior ocorrência durante o período de atividade física (35,2\%), seguida pelo repouso $(29,6 \%)$ e o período do dia em que ocorreram mais crises convulsivas foi pela manhã e noite (ambos com 14\%), entretanto, na maioria dos casos (32\%) o proprietário não soube informar. Lengweiler e Jaggy (1999) ao investigarem 25 cães da raça Golden retriever com El, também através de questionário respondido pelos proprietários, observaram que as crises convulsivas aconteceram geralmente durante o sono em dois terços dos cães. Jaggy et al. (1998) citando o artigo de Schwartz-Porsche
(1994), no qual afirmaram que as crises convulsivas ocorrem principalmente em casa, durante ou imediatamente após um período de repouso ou sono e raramente quando o animal está ativo, o que contrasta com nossas observações com respeito a maior ocorrência de crises em estado de atividade física. Na maioria dos casos $(28,6 \%)$, o proprietário não correlacionou a crise convulsiva com fatores desencadeantes, porém, em $19,6 \%$, a crise foi correlacionada com fatores estressantes. Quanto à história familiar, apenas em 6 casos $(12,0 \%)$ foi relatada. Três proprietários referiram o lado materno e três ao paterno. Nestes casos, as raças relacionadas foram o Pastor Alemão (2), Labrador (1), Cocker Spaniel (1) e Poodle (2). Com relação à classificação das crises convulsivas, houve um predomínio da forma primária generalizada com um total de 26 casos (52\%), seguida da forma parcial simples com generalização secundária (22\%) (Figura 3). Considerando 0 nível de consciência dos animais durante a crise convulsiva, verificou-se que a freqüência de casos com perda da consciência foi bastante elevada (92\%). Encontramos crises generalizadas em 48 cães (96\%), com a presença de característica tônica-clônica em 31 deles (64,6\%), seguidas por salivação e micção, estando de acordo com as observações feitas por Shell (1993) e Thomas (2000), quando estes afirmaram que as crises generalizadas tônicas-clônicas são as mais comuns observadas em cães epilépticos. Todas as fases das crises convulsivas foram observadas na amostra que estudamos, portanto discordante de Shell (1993), quando afirmou que o estágio de pródromo é raramente reconhecido em animais epilépticos. Entretanto, Knouwles (1998) e Thomas (2000) afirmaram que as fases de pródromo, aura e pós-ictus são comuns, especialmente em animais com crises epilépticas tônico-clônicas generalizadas. Dentre os sinais prodrômicos, a inquietação e a procura pelo dono foram os mais freqüentes, com um tempo de manifestação com variação de um até trinta minutos, resultado que está de acordo com Jaggy e Bernardini (1998), que encontraram a inquietação como sinal prodrômico mais freqüente, aparecendo em seguida medo, tremores, procura pelo dono e salivação. Verificamos dentre os sinais de aura parcial simples, o andar atáxico, vindo a seguir, como os mais freqüentes, a atonia dos membros pélvicos e o andar com o corpo curvado só para um lado. Dentre os sinais de aura parcial complexa, encontramos os sinais de confusão e a ausência de resposta a comandos como os mais citados pelos donos. A fase de aura com duração de até um minuto foi observada em $70 \%$ dos cães aproximadamente. Já Heynold et al. (1997) observaram uma duração de não mais que 30 segundos em suas investigações, duração que pode ser considerada como próxima da que encontramos. Em nosso estudo, os sinais pós-ictus que verificamos foram principalmente a desorientação, a hiperatividade e o andar compulsivo. O intervalo de um a cinco minutos de duração foi o mais observado, vindo logo em seguida o intervalo de mais de cinco minutos até uma hora. Comparativamente, Jaggy e Bernardini (1998) citaram fadiga, desorientação e andar compulsivo como os sinais mais encontrados na maioria dos cães que estudaram, registrando como mais freqüente quanto à duração, o intervalo de poucos minutos a duas ou mais horas.

Dos medicamentos utilizados antes da primeira consulta na UFRRJ, o mais empregado foi o fenobarbital (28 casos) seguido pelo diazepan (8 casos). A dose variou de 1,25 a 22,7 mg/ $\mathrm{kg}$ com uma mediana de $4,5 \mathrm{mg} / \mathrm{kg}$. Os efeitos colaterais mais relatados foram a polidipsia (13 casos), ganho de peso (13 casos), polifagia (11 casos) e poliúria (10 casos) (Figura 4). 


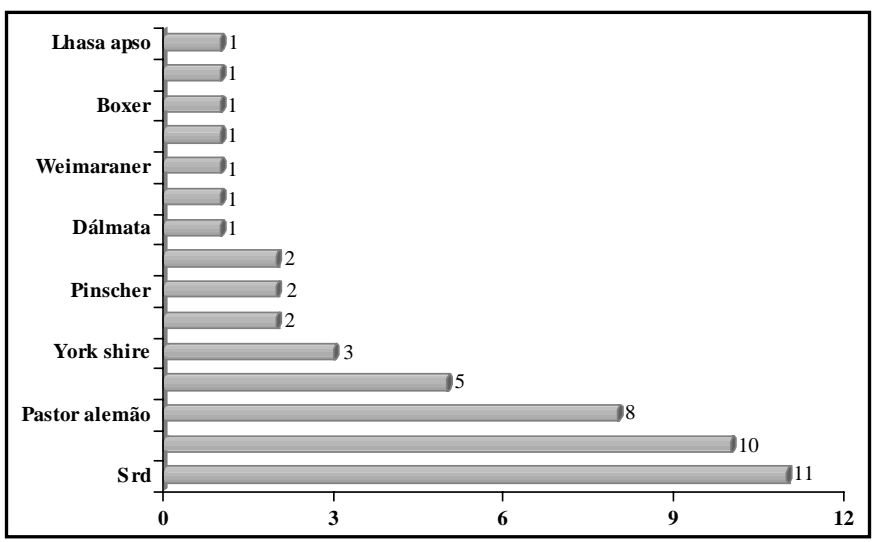

Figura 1: Distribuição de freqüências de Epilepsia Idiopática quanto à raça - Hospital Veterinário da UFRRJ - 2000-2002

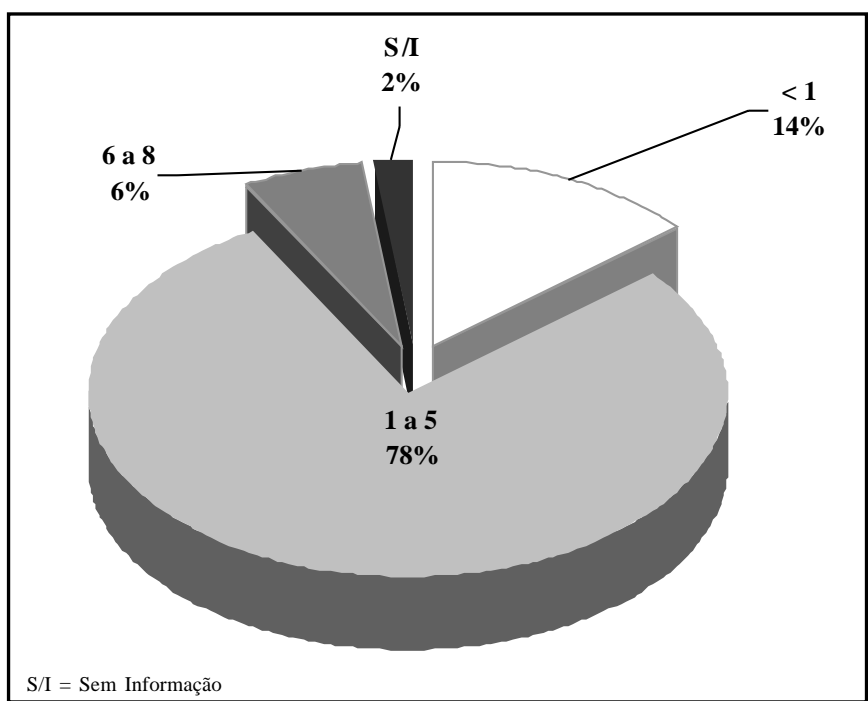

Figura 2: Distribuição de freqüências de Epilepsia Idiopática quanto à idade à primeira crise - Hospital Veterinário da UFRRJ - 20002002

O fenobarbital na dose de 2,0 a 5,0 mg/kg/BID mostrou-se bastante eficaz no controle das crises, sendo a dose aumentada ou diminuída quando necessário. Segundo Koutinas et al. (1994) a rotina de dosagem pode ficar entre 1,5 a $30 \mathrm{mg} / \mathrm{kg}$. Neste estudo, quatro cães (14,2\%) não tiveram mais crises convulsivas com o uso do fenobarbital. Saito et al. (2001) fazem referência ao fato de que 5 a $40 \%$ dos cães com El podem se tornar livres das crises convulsivas. Nossos resultados estão, portanto, em conformidade com tal afirmativa. Heynold et al. (1997), estudando cães da raça Labrador retriever com $\mathrm{El}$, encontraram melhores resultados no tratamento de cães onde houve um início tardio da doença (em média, quatro anos) e baixa freqüência de crises convulsivas anteriores à primeira consulta e ao início da terapia. Estes dois aspectos, segundo estes autores, explicariam a existência de grandes diferenças na resposta dos cães, embora todos estivessem sendo tratados seguindo o mesmo protocolo. Concluíram, portanto, pela necessidade de um início precoce da terapia médica, já que o sucesso desta tem relação direta com a rapidez com que é iniciada. Em nosso

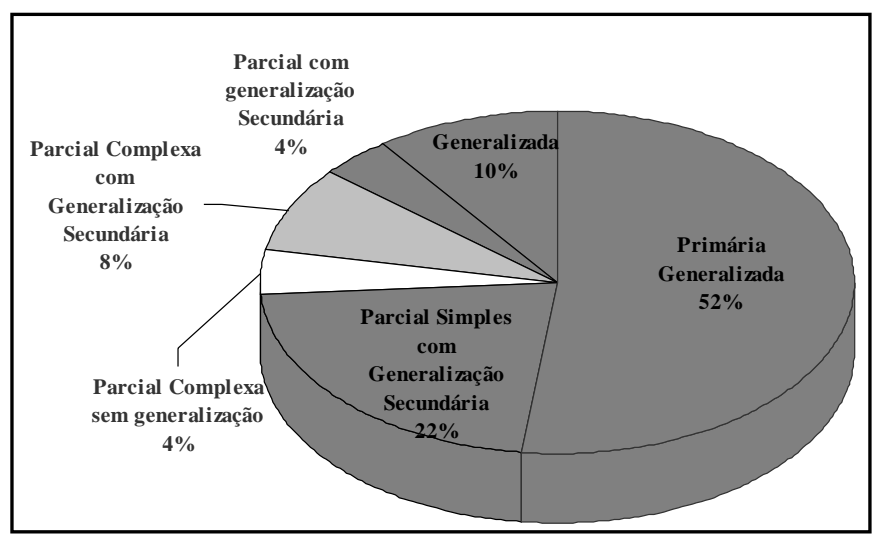

Figura 3: Distribuição de freqüências de Epilepsia Idiopática quanto à Classificação das Crises Convulsivas - Hospital Veterinário da UFRRJ - 2000-2002

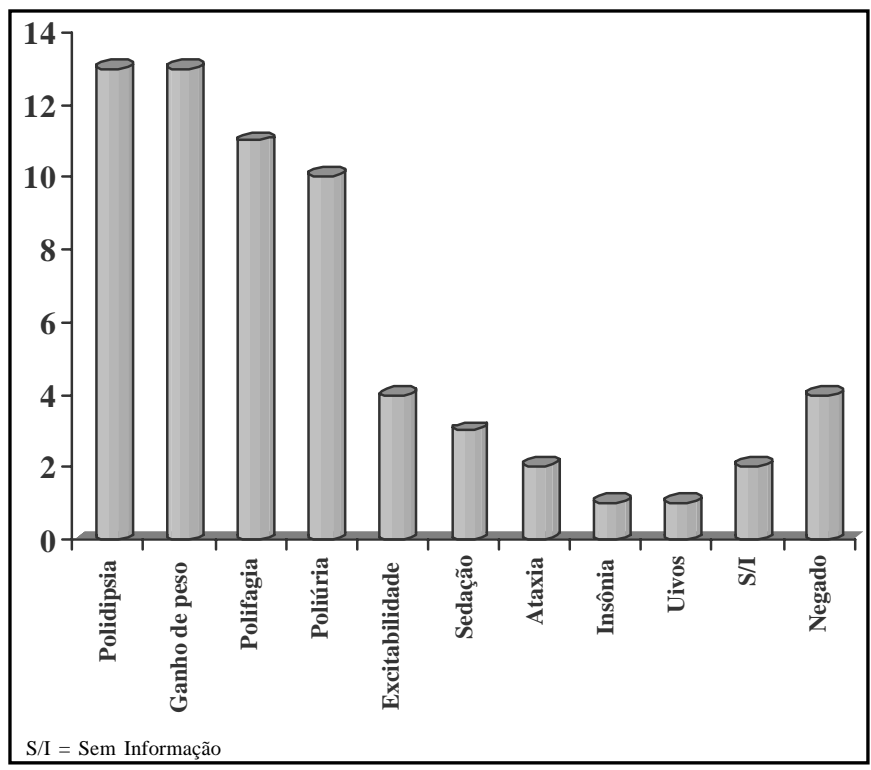

Figura 4: Efeitos colaterais mais relatados pelos proprietários de animais epilépticos durante o tratamento com fenobarbital

estudo, verificamos que $56 \%$ dos cães apresentaram de uma a três crises convulsivas antes da primeira consulta. Este foi um fator positivo em nosso inquérito, pois como grande parte dos animais teve poucas crises convulsivas antes do tratamento, há uma possibilidade de maior eficiência deste quando iniciado.

Neste estudo, o fenobarbital foi a droga mais utilizada como agente anticonvulsivante inicial, conforme foi verificado a partir da anamnese feita em consulta inicial com os proprietários de 28 animais (52\%). Este resultado está de acordo com Saito et al. (2001), que confirmaram o uso do fenobarbital como droga de primeira linha na medicação anticonvulsivante de cães com epilepsia, relatando sucesso no controle das crises em 60 a $80 \%$ dos cães com El. Constatamos em seis animais $(21,4 \%)$ um esquema de doses do fenobarbital uma vez ao dia. Um outro cão (3,5\%) apresentou administração da droga a cada 48 horas. Os demais 20 cães $(71,4 \%)$ estavam dentro do 
esquema padrão sugerido (12 em 12 horas) com dosagem adequada. Entretanto, todos estes animais continuaram apresentando crises, ora porque os períodos de administração da droga eram curtos (menores que duas semanas), ora porque tinham ganho de peso descompensando os níveis séricos sanguíneos, ora, ainda, porque apresentavam aumento do metabolismo hepático. Thomas (2000) citou como exemplos de erros comuns na terapia com fenobarbital as dosagens incorretas ou então a pouca obediência dos proprietários ao esquema proposto, quando este está correto. Este autor citou ainda, como uma causa comum para o pouco controle das crises, o uso de drogas distintas, uma em seguida a outra, dadas por tempo insuficiente ou então em doses abaixo do que seria recomendável. Selcer e Selcer (1990) também citaram como causas para o insucesso da terapia a dose insuficiente do anticonvulsivante e o intervalo entre as doses além das doze horas indicadas. Portanto, os dados encontrados neste trabalho estão de acordo com os autores citados. A excitabilidade e a sedação foram os efeitos colaterais cita-dos com menor freqüência pelos proprietários que entrevis-tamos, ou seja, foram pouco percebidos por eles. Entretanto, quando esta percepção ocorria, provocava muita preocupa-ção e ansiedade nos mesmos, que acabavam reduzindo a dosagem por conta própria, antes do período de estabiliza-ção dos níveis séricos da droga, nos casos da sedação, ou então interrompiam o tratamento, nos casos de excitação.

Koutinas et al. (1994) listaram os efeitos colaterais mais comuns em relação ao uso do fenobarbital, que incluem o apetite voraz, aumento do peso corporal, polidipsia, poliúria e sonolência. Em muitos casos, estes efeitos colaterais desapareceram em aproximadamente duas semanas após o início do tratamento. No estudo destes autores, eles foram verificados em todos os oito cães tratados, desaparecendo dentro de duas semanas, com a exceção de dois cães. Estes continuaram a ter aumento de apetite durante vários meses,

\section{Referências}

BATEMAN, S.; PARENT, J. M.. Clinical findings, treatment, and outcome of dogs with status epilepticus or cluster seizures: 156 cases (19901995). Journal of American Veterinary Medicine Association, v. 215, n. 10, p. 1463-1468, 15 nov. 1999.

BERENDT, M.; GRAM L.. Epilepsy and Seizure Classification in 63 Dogs:A Reappraisal of Veterinary Epilepsy Terminology. Journal of Veterinary Internal Medicine, v. 13, n. 1, p. 14-20, 1999.

BERENDT, M. et al.. A Cross-Sectional study of epilepsy in Danish Labrador Retrievers: Prevalence and selected risk factors. Journal Veterinary Intern Medical, v. 16, p. 262-268, 2002.

BOLLINGER-SCHMITZ, K.; KLINE, K. An overview of canine idiopathic epilepsy for the small animal practitioner. lowa State University Veterinarian, v. 62, n. 1, p. 22-27, spring 2000

FARMULA, T. R.; OBERBAUER, A. M. Reducing the incidence of epileptic seizures in the Belgian Tervuren through selection. Preventive Veterinary Medicine, v. 33, p. 251-259, 1998.

HEYNOLD, Y et al. Clinical, epidemiological and treatment results of idiopathic epilepsy in 54 labrador retrievers: a long-term study. Journal of Small Animal Pratice, v. 38, p. 7-13, jan. 1997 o que levou-os a uma obesidade severa. Em nosso estudo, os efeitos colaterais mais citados em relação ao fenobarbital - considerados de forma não excludente, ou seja, um mesmo animal pode ter apresentado mais de um desses efeitos foram o ganho de peso (13 animais), a polidipsia (13) e a polifagia (11). Quanto ao brometo de potássio, o ganho de peso nos animais foi o efeito colateral mais acentuado. É importante ressaltar que estes efeitos, mencionados como os mais freqüentes, permaneceram durante todo o estudo, tanto para o fenobarbital quanto para o brometo de potássio.

Três cães com crises a cada seis a oito meses, nos quais o tratamento não foi iniciado, estavam com um período de observação com um limite entre 8 meses e 2 anos, sem alteração na freqüência, na severidade e na duração das crises convulsivas.

O brometo de potássio foi utilizado em apenas dois cães (4\%) desde o início do tratamento, com bom controle das crises convulsivas. O período total de observação dos resultados de tratamento foi de dois anos para um deles e cinco anos e nove meses para o outro.

Em todos os animais estudados, os exames clínico e neurológico entre as crises convulsivas nada revelaram de anormal, assim como os exames complementares, sugerindo fortemente, tratarem-se de casos de Epilepsia Idiopática (Koutinas et al., 1994).

Concluímos que a epilepsia idiopática teve uma ocorrência maior nos animais SRD, machos e entre 1 e 5 anos de idade; que houve um maior número de crises convulsivas generalizadas do tipo tônico-clônica, com perda de consciência e em estado de atividade física; que não foram evidenciados fatores desencadeantes de convulsão pela maioria dos proprietários; que os tratamentos com feno-barbital e/ou brometo de potássio foram eficientes na redução da freqüência de crises convulsivas e que a polidipsia e ganho de peso foram os efeitos colaterais mais citados pelos proprietários neste inquérito.

JAGGY, A. et al. Genetic aspects of idiopathic epilepsy in Labrador retrievers. Journal of Small Animal Pratice, v. 39, p. 275-280, june 1998.

JAGGY, A.; BERNARDINI, M. Idiopathic epilepsy in 125 dogs: a longterm study. Clinical and electroencephalographic findings. Journal of Small Animal Pratice, v. 39, p. 23-28, jun. 1998.

KATHMANN, I.. et al.. Clinical and genetic investigations of idiopathic epilepsy in the Bernese mountain dog. Journal of Small Animal Pratice, v. 40, p. 319-325, 1999.

KNOWLES, K. Idiopathic Epilepsy. Clinical Techniques in Small Animal Practice, v. 13, n. 3, p. 144-151, aug. 1998.

KOUTINAS, A. F. et al. Clinical and clinicopathological evaluation in 14 dogs with presumed idiopathic epilepsy. Deltion tes Ellenikes Kteniatrikes Etaireias - Bulletin of the Hellenic Veterinary Medical Society, v. 45, n. 2, p. 141-149, 1994.

LENGWEILER, C.; JAGGY, A. Clinical, epidemiological and treatment aspects of idiopathic epilepsy in 25 Golden Retrievers: results of a long-term study. Schweizer-Archiv-fur-Tierheilkunde, v. 141, n. 5, p. 231-238, 1999.

PELLEGRINO, F. Epilepsia Y síndromes epilepticos em felinos. Selecciones Veterinarias, v. 7, n. 4, 1999. 
PODELL, M.; FERNNER, W.R. Bromide therapy in refractory canine idiopathic epilepsy. Journal of Veterinary Internal Medicine, v. 7, p. 318-327, 1993.

PODELL, M. The use of diazepam per rectum at home for the acute management of cluster seizures in dogs. Jounal of Veterinary Internal Medicine, v. 9, n. 2, p. 68-74, mar.-aprill 1995.

SAITO, M. et al.. Risk factores for development of status epilepticus in dogs with idiopathic epilepsy and effects of status epilepticus on outcome and survival time: 32 cases (1990-1996). Journal of Animal Veterinary Medical Association, v. 219, n. 5, p. 618-623, 1 sept. 2001.
SELCER, R. R.; SELCER, E. S. A practical approach to seizure managemente in dogs and cats. Progress in Veterinary Neurology, $v$. 1, n. 2, p. 147-156, 1990.

SHELL, L. G. Understanding the fundamentals of seizures. Veterinary Medicine, p. 622-628, July 1993.

THOMAS, W.B. Idiopathic epilepsy in dogs. Veterinary Clinics of North America: Small Animal Pratice, v. 30, n. 1, p. 183-205, jan. 2000. 